\title{
DISTRIBUCIÓN DE LA METENCEFALINA EN LA VÍA ÓPTICA. ESTUDIO EXPERIMENTAL INMUNOCITOQUÍMICO
}

\author{
DISTRIBUTION OF MET-ENKEPHALIN EXPRESSION IN THE \\ VISUAL PATHWAY. EXPERIMENTAL STUDY BY \\ INMUNOCYTOCHEMISTRY
}

\author{
MONTERO-DE-ESPINOSA I ${ }^{1}$, MÁRQUEZ-DE-ARACENA R ${ }^{1}$, MUÑOZ-SAEZ M ${ }^{1}$
}

\begin{abstract}
RESUMEN
Objetivo: Conocer la localización y distribución de neuropéptidos en la vía óptica, concretamente, la determinación de inmunorreactividad a met-encefalina, clave para la funcionalidad de dicha vía.

Método: Se analizó la vía óptica de ocho gatos. Tras extracción quirúrgica; cortes con microqueratomo y procesamiento mediante inmunocitoquímica indirecta, utilizando como anticuerpo la antimetencefalina, con el fín de detectar la presencia o ausencia del pentapéptido en la vía óptica del gato. Resultados: Se detectaron receptores a met-encefalina en áreas del encéfalo tanto corticales como subcorticales. Así, se considera la posible implicación de dicho pentapéptido en la funcionalidad de la vía óptica.

Conclusiones: La presencia de receptores a metencefalina en áreas del encéfalo tanto corticales como subcorticales, muestra la posible implicación de dicho neurotransmisor en la funcionalidad de la vía óptica.
\end{abstract}

Palabras clave: Sustancia P, vía óptica, neurotransmisor, neuroprotección.

\begin{abstract}
Purpose: The localization and distribution of neuropetide expression in the cat visual pathway can provide information about the function of that pathway. Method: Study of optic pathway in eight cats. Following extraction of the brain, slices were prepared using a microkeratome. The slices were examined by indirect immunocytochemistry using antimetenkephalin as antibody to determine the presence or absence of this pentapeptide in the visual pathway.

Results: Met-enkephalin receptors in both cortical and subcortical regions of the brain were detected. This suggests that met-enkephalin could be involved in the visual mechanism.

Conclusions: The presence of met-enkephalin receptors in both cortical and subcortical regions of the brain suggests that this pentapeptide could be involved in the visual mechanism (Arch Soc Esp Oftalmol 2009; 84: 245-250).
\end{abstract}

Key words: Substance P, optic nerve, visual pathway, neurotransmitter, neuroprotection.

\footnotetext{
Recibido: 31/5/07. Aceptado: 12/5/09.

Servicio de Oftalmología. Hospital Universitario Virgen del Rocío. Sevilla. España.

1 Doctor en Medicina.

Correspondencia:

Ignacio Montero de Espinosa

Docencia Pregrado. Edificio de Gobierno

Hospital Universitario Virgen del Rocío

C/. Manuel Siurot, s/n

41013 Sevilla

España

E-mail: eignacio.montero.sspa@juntadeandalucia.es / maria96@supercable.es
} 


\section{INTRODUCCIÓN}

Hace más de treinta años que se conocen los neuropéptidos y con ellos la neuromodulación. Estos no sólo liberan hormonas a la sangre, sino también forman sinapsis sobre la superficie de otras células. Los péptidos reúnen una serie de características que les diferencian de los neurotransmisores clásicos, como la activación de los receptores a concentraciones mucho más bajas y una duración mucho mayor que los neurotransmisores convencionales (1). Se caracterizan por la coexistencia: una misma sinapsis puede liberar dos o más neurotransmisores (peptídico y clásico) al mismo tiempo (1-3); y la co-transmisión: regulación de un neutransmisor peptídico o clásico por otro de naturaleza peptídica (4). Hasta ahora se han descrito más de 50 neuropéptidos, realizándose varias clasificaciones de los mismos.

La metionina-encefalina fue uno de los primeros opiáceos endógenos que se aisló, con propiedades analgésicas y euforizantes tipo morfina. Se caracteriza por su alta selectividad y estereoespecificidad (5). Cualquier cambio estructural las inactiva y pueden actuar como antagonistas opiáceos. Existe un elevado número de receptores en los vertebrados (4).

El mapa de distribución del receptor opiáceo en el cerebro coincide con la vía paleo-espinotalámica del dolor, encontrándose también en la amígdala, cuerpo estriado e hipotálamo. La variabilidad de localizaciones coincide con la diversidad de funciones. Su distribución coincide bastante con la de los receptores opiáceos tanto exógenos como endógenos, principalmente la encefalina. Los receptores opiáceos se dividen en receptores $\mu$, a su vez subdivididos en receptores delta y kappa (6).

La presencia de receptores para opiáceos en el cerebro hizo pensar que dichos receptores serían utilizados por determinadas sustancias endógenas, demostrándose la presencia de encefalinas en ratas (7). Posteriormente, se pudo comprobar que son neurotransmisores de sistemas neuronales específicos, localizados en el cerebro, que participan en la integración de la información sensorial relacionada con el dolor y el comportamiento emocional (8).

Los péptidos opiáceos derivan de la proopiomelanocortina (POMC), proencefalina (de la que deriva la met-encefalina) y de la prodinorfina (9).

La metencefalina es una molécula que actúa como neurotransmisor; es asimismo un neuromodulador, pues modula la acción de neurotransmisores clásicos mediante el fenómeno histológico de coe- xistencia y fisiológico de cotransmisión (1). También actúa como hormona (a distancia) teniendo actividad neuroendocrina, ya que actúa sobre la hipófisis modificando la liberación de algunas hormonas: estimulan la secreción de ACTH, GH y prolactina en el lóbulo anterior, de MSH en el intermedio y de $\mathrm{ADH}$ en el posterior; mientras que inhíben la secreción de TSH, LH y FSH. Así pues, basándonos en esta multifuncionalidad podemos considerar la metencefalina como una molécula con capacidad para interconectar diferentes sistemas cerebrales.

La metencefalina está implicada en diferentes comportamientos y fenómenos fisiológicos que comprenden desde la percepción y modulación del dolor a la respuesta al stress, aprendizaje, balance energético, actividad motora, alimentación, conducta sexual, sueño y regulación térmica y respiratoria $(4,12,15)$.

\section{SUJETOS, MATERIAL Y MÉTODO}

Se utilizó el quirófano experimental y animalario de nuestro centro.

Recurso experimental: Se utilizaron ocho gatos machos adultos de un peso comprendido entre $2,5-4 \mathrm{~kg}$.

Material de laboratorio y biológico: Anticuerpo 1 (anti-metionina encefalina); anticuerpo 2 (anti Ig $\mathrm{H}+\mathrm{L}$ de conejo unido a una peroxidasa); suero normal de carnero.

Material químico: suero fisiológico, paraformaldehido, tampón Sörensen, sacarosa, agua destilada, agua oxigenada, ácido clorhídrico, hidroxi-metilamino-metano (TRIS), tritón X-100, diaminobencidina (D.A.B.), glicerina.

Material de microscopía óptica, fotográfico y cartográfico (mapa del sistema nervioso central -SNC- del gato de Reinoso, F.).

Sobre ocho gatos se procedió, previa anestesia con ketamina, al abordaje quirúrgico del ventrículo izquierdo. Se efectuaron cortes de los globos oculares y de la masa encefálica (de un grosor aproximado de 80 micras) mediante un microqueratomo de congelación. Los cortes se efectuaron en base a un sistema estereotáxico centrado por un plano horizontal que contiene a ambos ejes tragoinfraorbitarios y por un plano coronal o frontal que porta el eje biauditivo, siendo el punto medio referido eje 0 estereotáxico del sistema, y el plano que lo contiene el plano 0 .

Se procesaron mediante inmunocitoquímica indirecta, utilizando como anticuerpo la anti-metence- 
falina con el fin de detectar la presencia o ausencia del pentapéptido.

Tras el revelado de la muestra con D.A.B. se lleva a cabo el montaje de las mismas y se realizó una clasificación por regiones.

La presencia de inmunorreactividad observada a nivel de las estructuras anatómicas se objetivaron mediante microscopio óptico y una vez determinada la localización e intensidad de la inmunorreactividad, extrapolamos los resultados a los mapas topográficos del SNC del gato de Reinoso (11), teniendo en cuenta que $1 \mathrm{~cm}$ en el mapa corresponde a $1 \mathrm{~mm}$. En ella se reflejan 50 cortes desde 30 en los anteriores $(\mathrm{A}+30)$ hasta 20 en los posteriores $(\mathrm{P}-$ 20), se representaron sólo los cortes en que se halló alguna inmunorreactividad (A+15 a P-10).

\section{RESULTADOS}

Los hallazgos inmunorreactivos fueron clasificados dependiendo de su intensidad: Baja, Moderada e Intensa.
Se han encontrado una inmunorreactividad baja en el cuerpo geniculado lateral, en el pulvinar, pretectum y corteza cerebral áreas 17, 18 y 19 (tabla I).

La intensidad ascendió a moderada en el colículo superior y en los estratos grises superficial, medio y profundo (tabla II).

No se encontró inmunorreactividad en las demás áreas de la vía óptica.

\section{DISCUSIÓN}

Se utilizó el gato como animal de experimentación por disponer de unos mapas cartográficos muy completos de este animal (9).

Se han descritos mapas cartográficos del cerebro de este mismo pentapéptido en el cerebro de distintos animales gracias a las técnicas de inmunocitoquímica, la misma que hemos utilizado en este estudio: en ratas (10), gatos, monos y humanos.

Es en el núcleo estriado, concretamente en el globus pallidus, donde se concentran las más altas den-

Tabla I. Inmunorreactividad a met-encefalina en la vía óptica del gato

\begin{tabular}{|c|c|c|c|}
\hline Inmunorreactividad & Moderada & Baja & Nula \\
\hline Anterioridad $\mathrm{A}+15$ & & Corteza: Áreas 18 y 19 & Q.O. \\
\hline Anterioridad $\mathrm{A}+14$ & & Corteza: Áreas 18 y 19 & Q.O. \\
\hline Anterioridad $\mathrm{A}+13$ & & Corteza: Áreas 18 y 19 & T.O. \\
\hline Anterioridad $\mathrm{A}+12$ & & Corteza: Áreas 18 y 19 & T.O. \\
\hline Anterioridad $\mathrm{A}+11$ & & Corteza: Áreas 18 y 19 & T.O. \\
\hline Anterioridad $\mathrm{A}+10$ & & Corteza: Áreas 18 y 19 & T.O. \\
\hline Anterioridad A+9 & & Corteza: Áreas 18 y 19 & T.O. \\
\hline Anterioridad $\mathrm{A}+8$ & & Corteza: Áreas 18-19, G.L., P. & R.O., G.M, T.O. \\
\hline Anterioridad $\mathrm{A}+7$ & & C.: Áreas 18-19, GLD, GLV, P & G.M., T.O. \\
\hline Anterioridad $A+6$ & & C: $18-19$, GLD, GLV, P. y PR. & R.O.,T.O,GM \\
\hline Anterioridad $\mathrm{A}+5$ & & Corteza: Áreas 18-19, G.L. y P. & G.M. \\
\hline Anterioridad $\mathrm{A}+4$ & C.S.,E.G.M.,E.G.P., E.G.S. & Corteza: Áreas 18 y 19, G.L. & G.M. \\
\hline Anterioridad $\mathrm{A}+3$ & C.S.,E.G.M.,E.G.P., E.G.S. & Corteza: Áreas 18 y 19 & \\
\hline Anterioridad $\mathrm{A}+2$ & C.S.,E.G.M.,E.G.P., E.G.S. & Corteza: Áreas 17, 18 y 19 & \\
\hline Anterioridad $\mathrm{A}+1$ & C.S.,E.G.M.,E.G.P., E.G.S. & Corteza: Áreas 17,18 y 19 & \\
\hline Anterioridad $\mathrm{A}+0$ & C.S. & Corteza: Áreas 17, y 18 & \\
\hline Anterioridad A-1 & & Corteza: Áreas 17, y 18 & \\
\hline Anterioridad A-2 & & Corteza: Áreas 17, y 18 & \\
\hline Anterioridad A-3 & & Corteza: Áreas 17, y 18 & \\
\hline Anterioridad A-4 & & Corteza: Áreas 17, y 18 & \\
\hline Anterioridad A-5 & & Corteza: Áreas 17, y 18 & \\
\hline Anterioridad A-6 & & Corteza: Áreas 17, y 18 & \\
\hline Anterioridad A-7 & & Corteza: Áreas 17, y 18 & \\
\hline Anterioridad A-8 & & Corteza: Áreas 17, y 18 & \\
\hline Anterioridad A-9 & & Corteza: Áreas 17, y 18 & \\
\hline Anterioridad A-10 & & Corteza: Áreas 17, y 18 & \\
\hline
\end{tabular}

Colículo Superior: C.S.; Corteza: Corteza; Stratum Griseum Medium: E.G.M.; Stratum Griseum Profundum: E.G.P.; Stratum Griseum Superficiale: E.G.S.; Geniculado Lateral: G.L.; Geniculado Lateral Pars Dorsalis: GLD; Geniculdao Lateral Pars Ventralis: GLV; Pretectum: PR; Pulvinar: P; Quiasma Óptico: Q.O.; Tracto Óptico: T.O. 
Tabla II. Localización de receptores de met-encefalina en la vía óptica del gato

\begin{tabular}{ll}
\hline Inmunorreactividad & $\begin{array}{l}\text { Cuerpo Geniculado Lateral (G) } \\
\text { Pulvinar (PL) } \\
\text { Pretectum (PTE) } \\
\text { Corteza Visual (Áreas 17,18 y 19) }\end{array}$ \\
\hline Inmunorreactividad & $\begin{array}{l}\text { Colículo Superior (CL) } \\
\text { Stratum Griseum Médium (SGM) } \\
\text { Stratum Griseum Profundum } \\
\text { Stratum Griseum Superficiale }\end{array}$ \\
\hline \hline
\end{tabular}

sidades de fibras y terminales encefalinérgicas en el SNC $(10,11)$.

Otros lugares donde se objetivó la existencia de met-ecefalina fueron: los somas del cuerpo estriado, núcleo hipotalámico supraóptico y paraventricular y núcleos del tronco cerebral; fibras del telencéfalo en ciertas regiones de la corteza, septum lateral, núcleo central de la amígdala y cuerpo estriado; en diencéfalo tálamo, hipotálamo y subtálamo; del mesencéfalo formación reticular, sustancia gris periacueductual (SGPA) y núcleo interpeduncular; del tronco del encéfalo formación reticular, núcleo del rafe, locus coeruleus, núcleo del tracto solitario, núcleo espinal del trigémino, núcleo coclear, núcleo parabraquial, núcleo comisural y núcleo de pares craneales. También se objetivó presencia de metencefalina en el tálamo del gato (10).

Respecto al fenómeno de coexistencia (1) en la vía óptica antes apuntada donde se objetivó la existencia de met-encefalina y teniendo en cuenta la coexistencia peptídica y no peptídica $(1,4)$, se evidenció su coexistencia con otro neurotransmisor clásico o no peptídico.

La met-encefalina o Substancia $\mathrm{P}$, es una molécula que actúa como neurotransmisor ya que cumple los requisitos exigidos para estos; es asimismo un neuro-modulador y también actúa como hormona teniendo actividad neuroendocrina ya que actúan sobre la hipófisis. La met-encefalina está implicada en diferentes comportamientos y fenómenos fisiológicos que comprenden desde la percepción y modulación del dolor a la respuesta al stress y el aprendizaje.

Algunos autores han encontrado met-encefalina en algunas estructuras de la vía óptica: En la retina de la tortuga (18) y en la de la salamandra (19); en el pretectum de la paloma (20); en el núcleo geniculado $(21,22)$ y colículo superior de la rata $(20,21)$; en el conejo (23) y en el gato más recientemente (24).
Parece que la Sustancia P, a través de las células amacrinas, interviene en el desarrollo y funcionamiento de la retina del conejo (28). Mediante técnicas de inmunorreactividad se ha demostrado la presencia de Sustancia P en la retina humana, concretamente en la capa de fibras nerviosas, pero no en la capa plexiforme interna. A través de las células amacrinas se le ha supuesto cierta relación en la formación del retinoblastoma humano $(28,30)$.

Tras objetivar la existencia de met-encefalina en determinadas estructuras de la vía óptica, se demostró su posible interrelación con otras estructuras de la vía óptica $(27,28)$, suponiéndose un papel en el tracto retino-hipotalámico relacionado con el ciclo de la luz y la oscuridad (29) y cierta actividad en el desarrollo y funcionalidad de la retina (25). En dicha situación, nos planteamos el papel que desempeñaría en su fisiología. Partiendo de la base de que dicho pentapéptido es un neurotransmisor inhibidor, podría inhibir la neurotransmisión de dicha vía desempeñando un papel en la regulación del impulso nervios en estas estructuras. La activación de las fibras del sistema óptico producida por agentes estimulantes físicos externos (estimulantes lumínicos) y/o químicos-endógenos se vería antagonizada o parcialmente inhibida.

Podría pensarse que la base bioquímica-funcional de una «visión agradable» pudiera estar relacionada con la liberación de met-encefalina liberada tras la percepción, la cual a través de una conexión directa con el sistema límbico produciría el fenómeno gratificante. Otro posible mecanismo sería que tras liberar met-encefalina en el líquido cefalorraquídeo y en sangre se estimulara indirectamente dicho sistema límbico. $\mathrm{O}$ en foma conjunta ambos mecanismos.

Otro aspecto a destacar es relativo a la existencia de miosis y midriasis tras la administración de opiáceos endógenos y a su abstinencia, respectivamente. Haber encontrado inmunorreactividad a las metencefalina en el Pretectum pudiera explicar, al menos en parte, este fenómeno ya que la vía desde la retina hasta el Pretectum es importante en el reflejo pupilar.

Puede entenderse una buen relación anatomofisiológica al objetivar inmunorreactividad frente a la met-encefalina tanto en el núcleo Pulvinar como en Áreas 18 y 19 de la Corteza Visual (áreas extraestriadas), es bien conocido que este complejo núcleo talámico se proyecta ampliamente hacia regiones corticales visuales extraestriadas y que muchas de estas regiones corticales se proyectan de forma recíproca hacia el Pulvinar (28). 
De los conocimientos acumulados sobre la presencia de metencefalina en la vía óptica se puede deducir que:

- La presencia del neurotransmisor inhibidor met-encefalina en la vía óptica del gato actuaría inhibiendo o enlenteciendo la neurotransmisión en dicha vía.

- La no inmunorreactividad a met-encefalina en algunos segmentos de la vía óptica no excluye que pudiera estar presente en dicha vía.

- La presencia de inmunorreactividad a metencefalina en la vía óptica del gato fundamenta neuro-histoquimicamente la funcionalidad de dicha vía.

- La existencia de met-encefalina en la vía óptica del gato podría contribuir a la utilización racional y científica de fármacos agonistas o antagonistas de dicho péptido en la patología de la vía óptica.

Se confirma la existencia de inmunorreactividad a met-encefalina en la vía óptica del gato, lo que implica a este pentapéptido en la funcionalidad de dicha vía.

La distribución del pentapétido met-encefalina en la vía óptica del gato se objetiva en todo el encéfalo: porción cortical y subcortical.

La presencia de met-encefalina en la vía óptica del gato contribuye a la mejor comprensión funcional y bioquímica de dicha vía.

\section{BIBLIOGRAFÍA}

1. Hokfelt T, Lundberg JM, Schultzberg GM, Johansson O, Ljungdahl A, Rehfeld, J. Coexistence of peptides and putative transmitters in neurons. Adv Biochem Psychopharmacol 1980; 22: 1-23.

2. Johansson O, Hokfelt T, Pernow B, Jeffcoate SL, White N, Steinbusch, HW, et al. Inmunohisotchemical support for three putative transmitters in one neuron: coexistence of 5hydroxitryptamine, substance $P$-and thyrotropin releasing hormone-like inmunoreactivity in medullary neurons projecting to the spinal cord. Neuroscience 1980; 6: 18571881 .

3. Leah JD, Cameron AA, Kelly WL, Snow PJ. Coexistence of peptide inmunoreactivity in sensory neurons of the cat. Neuroscience 1985; 16: 683-690.

4. Jansens KL, Faull RL, Dragunow M. Distribution of excitatory aminoacid, sigma, monoamina, catecholamine, acetilcolina, opioid, Substance P, adenosina and neuropeptide receptor in human motor and somatosensory cortex. Brain Res 1991 566: 225-238.

5. Goldstein A. Opioid peptides endorphins in pituitary and brain. Science 1976; 193: 1081-1086.

6. Paterson SJ, Robson LE, Kosterlitz HW. Classification of opioid receptors. Brain Med Bull 1983; 39: 31-36.
7. Hughes J. Isolation of an endogenous compound from the brain with pharmacological properties similar to morphine. Brain Res 1975; 88: 295-308.

8. Leboyer M. Endomorphines. Physiological and pharmacological aspects and research in psychiatry. Encephale 1986; 12: 45-62.

9. Rossier J. Enkephalin byosinthesis: the search for a commun precursor. Trends. Neuroscience 1981; 4: 94-97.

10. Miguel-Hidalgo JJ, Senba E, Takatsuji K, Tohyama M. Fine structure of synapses and retinal innervation of substance $P$ and adenosin deaminase containing neurons in the superior colliculus of the rat. J Comp Neurol 1991; 303: 63-74.

11. Reinoso-Suarez F. Topographischer Hirn Atlas der Katze. Ed. Merck A.G. Darmstadt, 1961.

12. Pickel VM, Sumal KK, Beckley SC, Miller RJ, Reis, DJ. Inmunocytochemical locatization of enkephalin in the neostriatum of rat brain: a light and electron microscopic study. J Comp Neurol 1980; 189: 721-740.

13. Somogyi P, Priestley JV, Cuello AC, Smith AD, Takagi, H. Synaptic connections of enkephalin-immunorreactive nerve terminals in the neostriatum: a correlated light an electron microscopic study. J Neurocytol 1982; 11: 779-807.

14. Vazquez J, Muñoz M, Coveñas R. Alterations on the distribution of methionine-enkephalin-like in the cat thalamus after electroacupunture: an immunocytochemical study. $J$ Hirnforsch 1990; 31: 555-561.

15. Akil H, Watson SJ, Young E, Lewis ME, Khachturian H, Walker JM. Endogenous opioids: biology and function. Annu Rev Neurosci 1984; 7: 223-255.

16. Weiler $R$. Mesencephalic pathway to the retina exhibits enkephalin-like immunoreactivity. Neurosci Lett 1985; 55: 11-16.

17. Glasener $G$, Himstedt W, Weiler R, Matute C. Putative neurotransmitters in the retinae of three urodele species (Triturus alpestris, Salamandra salamandra, Pleurodeles waltli). Cell Tissue Res 1988; 252: 317-328.

18. Reiner A, Karter HJ, Brecha NC. Enkephalin-mediated basal ganglia influences over the optic tectum: inmunohistochimestry of the tectum and the lateral spiriform nucleus in pigeon. J Comp Neurol 1982; 208: 37-53.

19. Abe J, Okamura H, Makino S, Yanaihara N, Ibata Y. Inmunocytochemical distribution of [Met]enkephalin-Arg-GlyLeu inmunoreactivity in the rat diencephalon. Brain Res Bull 1987; 19: 735-741.

20. Card JP, Moore RY. Organization lateral geniculate-hypothalamic connections in the rat. J Comp Neurol 1989; 284: 135-147.

21. Miguel-Hidalgo JJ, Senba E, Takatsujy K, Tohyama M. Substance $P$ and enkephalins in the superficial layers of the rat superior colliculus: diferential plastic effects of retinal deafferentation. J Comp Neurol 1990; 299: 389404.

22. Okamoto S, Okamura H, Akagi Y, Yanaihara N, Ibata Y. Possible induction of [Met]-enkephalin-Arg6-Gly7-Leu8 immunoreactivity in neurons of the rat superior colliculus following eye enucleation. Neurosci Lett 1990; 120: 74-79.

23. Vecino E, Ekstrom P. Distribution of Met-enkephalin, Leuenkephalin, substance P, neuropeptide Y, FMRFamide, and serotonin inmunoreactivities in the optic tectum of the Atlantic salmon (Salamo salar L). J Comp Neurol 1990; 299: 229-241. 
24. Illing RB, Nikolarakis KE, Wichmann T, Spatz WB, Starke $K$. Release of met-enkephalin and its modulation through acetylcholine receptors in the rabbit superior colliculus. Exp Brain Res 1990; 82: 663.

25. Vazquez, J, Muñoz M, Garcia JC. Modifications in the distribution of met-enkephalin in the pons of the cat, following the intravenous administration of clonidine: an inmunocytochemical experimental study. Histol Histopathol 1998; 13: 619-626.

26. Casini G, Dal Monte M, Fornai F, Bosco L, Willems D, Yang $Q$, et al. Neurokinin 1 receptor expression and substance $P$ physiological actions are developmentally regulated in the rabbit retina. Neuroscience 2004; 124: 147-160.

27. Tarkkanen A, Tervo T, Eranko L, Cuello AC. Substance P inmunorreactivity in normal human retina and in retinoblastoma. Ophthalmic Res 1983; 15: 300-306.

28. Miguel-Hidalgo JJ, Senba E, Takatsuji K, Tohyama M. Effects of the eye-enucleation on substance P-inmunorreactive fibers of some retinorecipient nuclei of the rat in relation to their origin from the superior colliculus. Neuroscience 1991; 44: 235-243.

29. Oyadama H, Takatsuji K, Senba E. Postnatal development NK1,NK2 and NK3 neurokin receptors expression in the rat retina. Brain Res.Dev Brain Res. 1999, 117: 59-70.

30. Muñoz M, Rosso M, Montero-Espinosa I. Neurokinin-1 receptors located in human retinoblastoma cell lines: antitumor action of its antagonist. Invest Ophthalmol Vis Sci 2007; 48: 2775-2781. 